\title{
Demonstrating that the nonorthogonal orbital optimized coupled cluster model converges to full configuration interaction
}

Rolf H. Myhre

Citation: The Journal of Chemical Physics 148, 094110 (2018); doi: 10.1063/1.5006160

View online: https://doi.org/10.1063/1.5006160

View Table of Contents: http://aip.scitation.org/toc/jcp/148/9

Published by the American Institute of Physics

\section{Articles you may be interested in}

Koopmans' theorem in the Hartree-Fock method. General formulation

The Journal of Chemical Physics 148, 094101 (2018); 10.1063/1.5019330

Lowering of the complexity of quantum chemistry methods by choice of representation

The Journal of Chemical Physics 148, 044106 (2018); 10.1063/1.5007779

Properties of coupled-cluster equations originating in excitation sub-algebras

The Journal of Chemical Physics 148, 094104 (2018); 10.1063/1.5010693

On the difference between variational and unitary coupled cluster theories

The Journal of Chemical Physics 148, 044107 (2018); 10.1063/1.5011033

Perspective: Ab initio force field methods derived from quantum mechanics

The Journal of Chemical Physics 148, 090901 (2018); 10.1063/1.5009551

Reduced-cost second-order algebraic-diagrammatic construction method for excitation energies and transition moments

The Journal of Chemical Physics 148, 094111 (2018); 10.1063/1.5021832

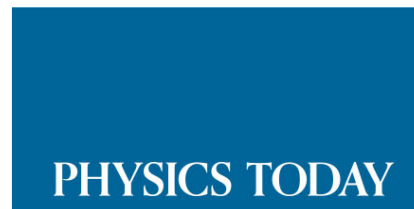




\title{
Demonstrating that the nonorthogonal orbital optimized coupled cluster model converges to full configuration interaction
}

\author{
Rolf H. Myhre ${ }^{a)}$ \\ Hylleraas Centre for Quantum Molecular Sciences, Department of Chemistry, University of Oslo, \\ 0315 Oslo, Norway
}

(Received 22 September 2017; accepted 19 February 2018; published online 7 March 2018)

\begin{abstract}
Coupled cluster $(\mathrm{CC})$ methods are among the most accurate methods in quantum chemistry. However, the standard CC linear response formulation is not gauge invariant, resulting in errors when modelling properties like optical rotation and electron circular dichroism. Including an explicit unitary orbital rotation in the CC Lagrangian makes the linear response function gauge invariant, but the resulting models are not equivalent to full configuration interaction (FCI) in the untruncated limit. In this contribution, such methods are briefly discussed and it is demonstrated that methods using a nonorthogonal orbital transformation, such as nonorthogonal orbital optimized CC, can converge to FCI in the untruncated limit. This has been disputed in the literature. Published by AIP Publishing. https://doi.org/10.1063/1.5006160
\end{abstract}

\section{INTRODUCTION}

Coupled cluster (CC) theory is the most accurate tool in regular use for describing molecular systems with an electronic wavefunction dominated by a single reference determinant. ${ }^{1}$ Such systems include most molecules in their ground state minimum energy geometry. The method can also be used to describe electronically excited states using the linear response ${ }^{2,3}$ (LR) or closely related equation of motion $^{4}(\mathrm{EOM})$ formalism. Due to the high accuracy of the model, current research is focused on reducing its relatively high computational cost and expanding it to systems with multireference character. Efforts in the former have focused on exploiting the short range of electron correlation to reduce the scaling of the $\mathrm{CC}$ models and have been quite successful. ${ }^{5-8}$ Many models have also been proposed to solve the multireference problem, but they tend to suffer from very high computational cost, instabilities, or low accuracy. ${ }^{9}, 10$

Another issue that has received less attention in the literature is the fact that standard truncated CC is not gauge invariant, even in the complete basis limit. ${ }^{11}$ This is a consequence of truncated CC not satisfying the conditions of the Ehrenfest theorem, Eq. (1), and results in discrepancies in properties like transition moments when using different gauges, for example, dipole length and dipole velocity,

$$
\frac{d}{d t}\langle A\rangle=i\langle[H, A]\rangle+\left\langle\frac{\partial A}{\partial t}\right\rangle .
$$

Typically, the discrepancies are quite small if the method provides a reasonable good description of the wavefunction and the basis set is sufficiently large. ${ }^{12}$ However, for properties depending on magnetic fields such as optical rotation

a)Email: r.h.myhre@kjemi.uio.no
(OR) and electron circular dichroism, translation in space is a gauge transformation. Consequently, the results of such calculations will depend on the placement of the origin in the dipole length gauge which is completely unphysical. ${ }^{13,14}$ In the dipole velocity gauge, CC OR calculations are origin independent but include an unphysical zero-frequency contribution. In the modified velocity gauge, the zero-frequency contribution is subtracted, resulting in consistent results at the cost of an additional calculation. ${ }^{15}$ Note that basis set incompleteness is also a cause of gauge dependence, but this can be avoided using a gauge including atomic orbitals. ${ }^{16,17}$

While standard CC theory does not satisfy the conditions of the Ehrenfest theorem, ${ }^{11}$ this can be achieved for one-electron operators by including an explicit orbital transformation in the CC Lagrangian. ${ }^{18,19}$ In standard CC, the right hand singles cluster operator, $T_{1}$, acts as an approximate orbital transformation ${ }^{20}$ and is removed when including an explicit orbital transformation because it is redundant. However, it is less clear what to do with the left hand singles cluster operator $\Lambda_{1}$, and several methods have been proposed. Note that for single reference systems, the orbital transformations are small and only result in small changes in total energy. However, for some systems where multireference character is caused by orbital instabilities, significant improvements can be observed. ${ }^{10,21}$

Brueckner $\mathrm{CC}^{22-24}$ (BCC) was, like standard $\mathrm{CC},{ }^{25}$ originally developed in nuclear physics. Orbital rotation parameters are included in the BCC Lagrangian exponentially, ensuring unitary transformations and orthogonal orbitals. The solution to the BCC equations is a wavefunction that is invariant with respect to the cluster amplitudes, and the orbitals are rotated to a basis where the singles right hand cluster operator is zero. The resulting model satisfies the Ehrenfest theorem, but unphysical second order poles appear in the response function. This and the fact that only small improvements are observed for the ground state energy 
compared to standard CC have limited the application of this model.

Orbital optimized coupled cluster ${ }^{18,21,26}$ (OCC) is similar to $\mathrm{BCC}$ and is also referred to as variational Brueckner CC. In this method, both the left and right hand singles amplitudes are set to zero and the orbital parameters are obtained by minimizing the energy. In this way, one obtains a response function with the correct pole structure. However, as pointed out by Köhn and Olsen, ${ }^{27}$ this method is not equivalent to full configuration interaction (FCI) in the untruncated limit. Nonetheless, the advantages of OCC have led to continued development of $\mathrm{CC}$ models with orbital optimization. For example, Crawford et al. proposed a CC model that combined orbital optimization with orbital localization in order to reduce the scaling of $\mathrm{CC}$ calculations for OR. ${ }^{28}$ In perfect paired CC, only cluster amplitudes involving paired electrons are retained. ${ }^{29}$ This greatly reduces the number of amplitudes and computational cost. Scuseria et al. combined the formalism with orbital optimization and demonstrated that the method gave good results, even for strongly correlated systems where standard CC fails. ${ }^{30,31}$ Recently, Head-Gordon et al. expanded the model to include paired quadruples and even hextuples in an active space. ${ }^{32,33}$ Note that the accuracy was improved when including singles in the cluster operator.

The nonorthogonal orbital optimized CC (NOCC) approach is similar to OCC in which both sets of singles amplitudes are redundant and set to zero. ${ }^{19}$ As implied by the name, the difference lies in the orbital transformation. By relaxing the demand for a unitary transformation, the orbitals are no longer orthogonal, but biorthogonal, resulting in a bivariational Lagrangian. ${ }^{34}$ In their paper, Köhn and Olsen conjectured that NOCC would suffer the same defects as OCC and not reach the FCI limit due to the lack of singles amplitudes. In this contribution, we will demonstrate that this is not the case and untruncated NOCC is equivalent to FCI.

Two other methods are worth a brief mention before we proceed. In the extended CC (ECC) $)^{35,36}$ method by Arponen, both the excitations and deexcitations are parametrized exponentially. Arponen showed that the standard CC model can be viewed as an approximation to ECC where the exponential of the deexcitations has been replaced by a linear parametrization that becomes identical in the FCI limit. The ECC Lagrangian is fully bivariational, ensuring the uniqueness and existence of a solution as well as bounds for the error. ${ }^{37}$ Unfortunately, this formulation results in an enormous number of terms, making a working implementation unfeasible. Another method based on the bivariational approach is the orbital adapted CC (OACC) method by Kvaal. ${ }^{38}$ This method is similar to NOCC, but the left and right-hand side orbitals are allowed to span different subspaces of the total orbital space, further increasing flexibility.

\section{NOCC EQUATIONS}

Nonorthogonal OCC is differentiated from standard OCC by the use of a biorthogonal basis instead of an orthogonal basis, and we will start our discussion with the rotational orbital parametrization. All expressions are in the spin orbital basis, and we only consider the untruncated FCI limit.
In order to ensure a unitary transformation, an exponential parametrization is employed in OCC. The orthogonal reference creation, $\hat{a}_{p}^{\dagger}$, annihilation, $\hat{a}_{p}$, operators and reference state function $|\hat{\phi}\rangle$ are transformed according to the following equation:

$$
\begin{aligned}
a_{p}^{\dagger} & =\exp (-\boldsymbol{\kappa}) \hat{a}_{p}^{\dagger} \exp (\boldsymbol{\kappa}), \\
a_{p} & =\exp (-\boldsymbol{\kappa}) \hat{a}_{p} \exp (\boldsymbol{\kappa}), \\
|\phi\rangle & =\exp (-\boldsymbol{\kappa})|\hat{\phi}\rangle .
\end{aligned}
$$

By demanding that $\kappa$ is antihermitian, the resulting transformation is unitary,

$$
\boldsymbol{\kappa}=\sum_{p q} \kappa_{p q} a_{p}^{\dagger} a_{q}, \quad \boldsymbol{\kappa}=-\boldsymbol{\kappa}^{\dagger} .
$$

It can be shown that rotations between two occupied or two virtual orbitals in the reference wavefunction are redundant in OCC, so only the off-diagonal blocks corresponding to occupied-virtual and virtual-occupied rotations are included in $\boldsymbol{\kappa} .^{18}$

In NOCC, the requirement that $\boldsymbol{\kappa}$ is antihermitian is removed, resulting in a non-unitary transformation of the orbitals. Equation (2) is still valid, but the creation and annihilation operators are no longer each other's complex conjugates. To emphasize this, we will label the annihilation operator and left-hand side with a tilde,

$$
\begin{aligned}
\left(\tilde{a}_{p}\right)^{\dagger} & =\left(\exp (-\boldsymbol{\kappa}) \hat{a}_{p} \exp (\boldsymbol{\kappa})\right)^{\dagger} \\
& =\exp \left(\boldsymbol{\kappa}^{\dagger}\right) \hat{a}_{p}^{\dagger} \exp \left(-\boldsymbol{\kappa}^{\dagger}\right) \\
& \neq \exp (-\boldsymbol{\kappa}) \hat{a}_{p}^{\dagger} \exp (\boldsymbol{\kappa})=a_{p}^{\dagger} .
\end{aligned}
$$

Despite not being conjugates of each other, the anticommutation relations hold and we can employ Wick's theorem in the standard way. ${ }^{39,40}$ Occupied-occupied and virtual-virtual orbital rotations are also still redundant, and we label the excitation and deexcitation parameters in $\boldsymbol{\kappa}$ with $u$ for up and $d$ for down for convenience. We use the standard notation where indices $i, j, k, \ldots$ and $a, b, c, \ldots$ refer to occupied and virtual orbitals in the reference state, respectively,

$$
\kappa=\sum_{a i} \kappa_{a i}^{u} a_{a}^{\dagger} \tilde{a}_{i}+\kappa_{i a}^{d} a_{i}^{\dagger} \tilde{a}_{a}=\sum_{a i} \kappa_{a i}^{u} X_{a i}+\kappa_{i a}^{d} Y_{i a} .
$$

In Eq. (5), we have introduced the right hand, $X_{a i}$, and left hand, $Y_{i a}$, singles excitation operators. Higher excitation operators are similarly defined, analogously to standard CC theory. In an orthogonal basis, these are each other's complex conjugates, but this is not generally true in a biorthogonal basis, $Y_{\mu} \neq X_{\mu}^{\dagger}$, and we need different symbols for the operators.

The starting point for the NOCC model is the bivariational NOCC Lagrangian $L$,

$$
\begin{aligned}
L & =\langle\tilde{\Psi}|H| \Psi\rangle \\
& =\langle\tilde{\phi}|(1+\Lambda) \exp (-T) \exp (-\boldsymbol{\kappa}) H \exp (\boldsymbol{\kappa}) \exp (T)| \phi\rangle .
\end{aligned}
$$

Explicitly including the orbital transformation terms in the derivation of the NOCC equations would result in extremely complicated expressions because $\boldsymbol{\kappa}$ does not commute with $T$ or $\Lambda$. We therefore express the equations in the optimized basis where $\boldsymbol{\kappa}=\mathbf{0}$, and a solution to the Schrödinger equation corresponds to a stationary point of the Lagrangian. From an implementation perspective, this can be viewed as expanding the 
exponentials of $\boldsymbol{\kappa}$ and only keeping zero order terms. This suggests an algorithm that iterates between orbital transformation and amplitudes until self-consistency. ${ }^{26}$

The exponential parametrization of the orbital rotations ensures that our basis and manifold of states are biorthogonal, and we assume a unit overlap between the reference bra and ket states,

$$
\begin{gathered}
\langle\tilde{\mu} \mid \phi\rangle=\left\langle\tilde{\phi}\left|Y_{\mu}\right| \phi\right\rangle=0, \quad\langle\tilde{\phi} \mid \mu\rangle=\left\langle\tilde{\phi}\left|X_{\mu}\right| \phi\right\rangle=0, \\
\langle\tilde{\phi} \mid \phi\rangle=1, \quad\langle\tilde{\mu} \mid v\rangle=\delta_{\mu, \nu} .
\end{gathered}
$$

$T$ and $\Lambda$ are defined similarly to the standard cluster operators except that the redundant singles excitations have been omitted,

$$
T=\sum_{\mu_{n}} \tau_{\mu_{n}} X_{\mu_{n}}, \quad \Lambda=\sum_{\mu_{n}} \lambda_{\mu_{n}} Y_{\mu_{n}}, \quad n \geq 2 .
$$

In Eq. (9), $\tau_{\mu}$ and $\lambda_{\mu}$ are the amplitude parameters of the operators. The NOCC left, $\langle\tilde{\Psi}|$, and right, $|\Psi\rangle$, wavefunctions must satisfy the standard CC equations, including the singles projection, in the biorthogonal basis in order to be eigenfunctions of the Hamiltonian. Note that the Hamiltonian is not Hermitian in this basis,

$$
\begin{aligned}
\langle\tilde{\mu}|\exp (-T) H| \Psi\rangle & =E\langle\tilde{\mu}|\exp (-T)| \Psi\rangle \\
& =E\langle\tilde{\mu}|\exp (-T) \exp (T)| \phi\rangle=E\langle\tilde{\mu} \mid \phi\rangle=0,
\end{aligned}
$$

$$
\begin{aligned}
\left\langle\tilde{\Psi}\left|\left[H, Y_{\mu}\right]\right| \Psi\right\rangle & =\left\langle\tilde{\Psi}\left|H Y_{\mu}\right| \Psi\right\rangle-\left\langle\tilde{\Psi}\left|Y_{\mu} H\right| \Psi\right\rangle \\
& =E\left(\left\langle\tilde{\Psi}\left|Y_{\mu}\right| \Psi\right\rangle-\left\langle\tilde{\Psi}\left|Y_{\mu}\right| \Psi\right\rangle\right)=0 .
\end{aligned}
$$

At the stationary point, the differential of $L$ must be zero with respect to the four sets of parameters: $\{\tau\},\{\lambda\},\left\{\kappa^{u}\right\}$, and $\left\{\kappa^{d}\right\}$, resulting in four sets of equations,

$$
\begin{aligned}
\frac{\partial L}{\partial \lambda_{\mu_{n}}} & =\left\langle\tilde{\mu}_{n}|\exp (-T) H \exp (T)| \phi\right\rangle, \\
\frac{\partial L}{\partial \tau_{\mu_{n}}} & =\left\langle\tilde{\phi}\left|(1+\Lambda) \exp (-T)\left[H, X_{\mu_{n}}\right] \exp (T)\right| \phi\right\rangle, \\
\frac{\partial L}{\partial \kappa_{\mu_{1}}^{u}} & =\left\langle\tilde{\phi}\left|(1+\Lambda) \exp (-T)\left[H, X_{\mu_{1}}\right] \exp (T)\right| \phi\right\rangle, \\
\frac{\partial L}{\partial \kappa_{\mu_{1}}^{d}} & =\left\langle\tilde{\phi}\left|(1+\Lambda) \exp (-T)\left[H, Y_{\mu_{1}}\right] \exp (T)\right| \phi\right\rangle .
\end{aligned}
$$

In order to prove that NOCC is equivalent to FCI, we must first demonstrate that the above equations result in a wavefunction that satisfies all the projection equations. In particular, the singles projection equation must be satisfied. ${ }^{27}$ Furthermore, for the equivalence to go both ways, we must prove that the standard CC wavefunction can be rotated to a basis where it satisfies the NOCC equations.

Equations (12) and (13) are the standard projection equations from $\mathrm{CC}$ theory and are required in order to satisfy the FCI equation. Similarly, Eq. (14) is identical to the derivative with respect to the right hand singles amplitudes in standard CC. Only Eq. (15) differs from the equivalent projection equations in standard $\mathrm{CC}$ and requires further analysis. To simplify the analysis, we will introduce some additional notations,

$$
\begin{aligned}
\langle\tilde{\Lambda}| & =\langle\tilde{\phi}|(1+\Lambda), \\
\tilde{H} & =\exp (-T) H \exp (T) .
\end{aligned}
$$

Using the Baker-Campbell-Hausdorff expansion and commuting out the $Y_{\mu_{1}}$-operator, we are left with three terms,

$$
\begin{aligned}
\frac{\partial L}{\partial \kappa_{\mu_{1}}^{d}}= & \left\langle\tilde{\Lambda}\left|\left[\tilde{H}, Y_{\mu_{1}}\right]\right| \phi\right\rangle+\left\langle\tilde{\Lambda}\left|\left[\tilde{H},\left[Y_{\mu_{1}}, T\right]\right]\right| \phi\right\rangle \\
& +\frac{1}{2}\left\langle\tilde{\Lambda}\left|\left[\tilde{H},\left[\left[Y_{\mu_{1}}, T\right], T\right]\right]\right| \phi\right\rangle
\end{aligned}
$$

We analyze the expression in Eq. (18) term by term and start with the first. Writing out the commutator, we get the standard singles projection with some additional terms,

$$
\left\langle\tilde{\Lambda}\left|\left[\tilde{H}, Y_{\mu_{1}}\right]\right| \phi\right\rangle=-\left\langle\tilde{\mu}_{1}|\tilde{H}| \phi\right\rangle-\sum_{n} \lambda_{\mu_{n}}\left\langle\tilde{\mu}_{n}\left|Y_{\mu_{1}} \tilde{H}\right| \phi\right\rangle .
$$

In the FCI limit, any projection against doubles and higher is zero due to Eq. (12) and Eq. (19) reduces to the standard singles projection equation. Similarly, the double commutator in the last term reduces to a modified cluster operator of triple excitations and higher,

$$
\left\langle\tilde{\Lambda}\left|\left[\tilde{H},\left[\left[Y_{\mu_{1}}, T\right], T\right]\right]\right| \phi\right\rangle=\sum_{\mu_{n}} B_{\mu_{n}}\left\langle\tilde{\Lambda}\left|\left[\tilde{H}, X_{\mu_{n}}\right]\right| \phi\right\rangle .
$$

Above, the coefficients $B_{\mu_{n}}$ are all linear combinations of products of two cluster amplitudes, and all these terms are zero due to Eq. (13).

The second term in Eq. (18) results in three types of terms,

$$
\begin{aligned}
& \pm\left\langle\tilde{\Lambda}\left|\left[\tilde{H}, X_{\mu_{n-1}}\right]\right| \phi\right\rangle, \\
& +\left\langle\tilde{\Lambda}\left|\left[\tilde{H}, X_{\mu_{n-1}} a_{b}^{\dagger} \tilde{a}_{a}\right]\right| \phi\right\rangle, \\
& +\left\langle\tilde{\Lambda}\left|\left[\tilde{H}, X_{\mu_{n-1}} \tilde{a}_{j} a_{i}^{\dagger}\right]\right| \phi\right\rangle .
\end{aligned}
$$

The sign of the first term depends on the order of the creation and annihilation operators, but this term is zero anyway due to Eqs. (13) and (14) and we only need to worry about the last two terms. When acting on the reference state, the extra creation and annihilation operators become zero, so we only need the terms with the operator to the left of the Hamiltonian. When including the cluster operator with amplitudes, the single commutator term in Eq. (18) takes the form in Eqs. (21) and (22). The compound index $\mu_{n+1}$ differs from $\mu_{n}$, in which it includes an extra excitation involving one external index,

$$
\begin{gathered}
\sum_{v_{m}, \mu_{n}, b} \lambda_{v_{m}} \tau_{\mu_{n+1}}\left\langle\tilde{\phi}\left|Y_{v_{m}} X_{\mu_{n}} a_{b}^{\dagger} \tilde{a}_{a} \tilde{H}\right| \phi\right\rangle, \\
\sum_{v_{m}, \mu_{n}, j} \lambda_{v_{m}} \tau_{\mu_{n+1}}\left\langle\tilde{\phi}\left|Y_{v_{m}} X_{\mu_{n}} \tilde{a}_{j} a_{i}^{\dagger} \tilde{H}\right| \phi\right\rangle .
\end{gathered}
$$

Equations (21) and (22) give different results depending on the excitation level of $v_{m}$ and $\mu_{n}$, and there are three different cases: $m \leq n, m=n+1$, and $m>n+1$. In the first case, the term is zero due to the projection and the last case is zero due to Eq. (12). When $m=n+1$, the term becomes a linear combination of the single projections with one index differing from the original external indexes,

$$
\left\langle\tilde{\Lambda}\left|\left[\tilde{H},\left[a_{i}^{\dagger} \tilde{a}_{a}, T\right]\right]\right| \phi\right\rangle=-\sum_{j} C_{j}^{a i}\left\langle{ }_{j}^{\tilde{a}}|\tilde{H}| \phi\right\rangle-\sum_{b} C_{b}^{a i}\left\langle{ }_{i}^{\tilde{b}}|\tilde{H}| \phi\right\rangle .
$$


The coefficients $C$ are products of the $\lambda$ amplitudes and antisymmetrized cluster amplitudes $\tau_{\mu_{n}, i}^{A S}$,

$$
C_{j}^{a i}=\sum_{\mu_{n, j}} \lambda_{\mu_{n, j}} \tau_{\mu_{n, i}}^{A S}, \quad C_{b}^{a i}=\sum_{\mu_{n, b}} \lambda_{\mu_{n, b}} \tau_{\mu_{n, a}}^{A S}
$$

Compound indexes of the type $\mu_{n, p}$ indicate that the excited state involves the orbital $p$ and the indices $\mu_{n, p}$ and $\mu_{n, q}$ differ only in this index. For example, the doubles contributions are sums over three indices,

$$
\begin{aligned}
C_{j}^{a i} & \leftarrow \sum_{b c k} \lambda_{j k}^{b c}\left(\tau_{i k}^{b c}-\tau_{i k}^{c b}\right)=2 \sum_{b c k} \lambda_{j k}^{b c} \tau_{i k}^{b c}, \\
C_{b}^{a i} & \leftarrow \sum_{c j k} \lambda_{j k}^{b c}\left(\tau_{j k}^{a c}-\tau_{k j}^{a c}\right)=2 \sum_{c j k} \lambda_{j k}^{b c} \tau_{j k}^{a c} .
\end{aligned}
$$

Adding the terms together, Eq. (15) reduces to the standard single projection and sums of single projections that differ in one index,

$$
\left.\frac{\partial L}{\partial \kappa_{i a}^{d}}\right|_{\kappa=\mathbf{0}}=-\left\langle{ }_{i}^{\tilde{a}}|\tilde{H}| \phi\right\rangle-\sum_{j} C_{j}^{a i}\left\langle\left\langle_{j}^{\tilde{a}}|\tilde{H}| \phi\right\rangle-\sum_{b} C_{b}^{a i}\left\langle{ }_{i}^{\tilde{b}}|\tilde{H}| \phi\right\rangle .\right.
$$

Equation (27) can also be written in matrix form

$$
\begin{gathered}
\mathbf{0}=\boldsymbol{A} \boldsymbol{x}, \\
x_{a i}=\left\langle\left\langle_{i}^{\tilde{a}}|\tilde{H}| \phi\right\rangle,\right. \\
A_{a i, b j}=\delta_{a i, b j}+\delta_{i, j} C_{b}^{a i}+\delta_{a, b} C_{j}^{a i} .
\end{gathered}
$$

The structure of $\boldsymbol{A}$ is visualized in Eq. (31), where $\times$ indicates a nonzero element of the matrices,

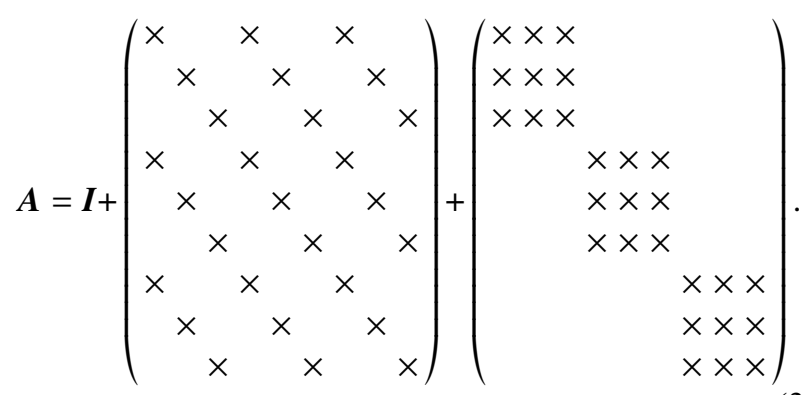

Clearly, a solution which satisfies all the singles projections, i.e., $\boldsymbol{x}=\mathbf{0}$, will satisfy Eq. (28), so $|\Psi\rangle$ will satisfy the NOCC equations if it is an eigenfunction of $H$. However, we also have to show that it is unique. In order for a matrix equation like Eq. (28) to have a unique solution, $\boldsymbol{A}$ must be nonsingular with $\operatorname{det}(\boldsymbol{A}) \neq 0$. If the FCI wavefunction is dominated by a single determinant, the amplitudes of $T$ and $\Lambda$ will be small. Consequently the off-diagonal elements in $\boldsymbol{A}$ are much smaller than 1 and $\boldsymbol{A}$ will be strictly diagonally dominant. Such matrices are never singular, and the solution to the NOCC equations is unique.

In the multireference case, we can no longer assume that the amplitudes are small. However, we first note that the space of singular $\boldsymbol{A}$-matrices is one dimensional because such matrices must satisfy $\operatorname{det}(\boldsymbol{A})=0$ and a minuscule change in any coefficient would make it nonsingular. Consequently, the chance of generating a singular matrix by choosing the coefficients at random is zero, given infinite numerical accuracy. In our case, the coefficients are not chosen at random but fixed by Eqs. (12) and (13). However it seems highly unlikely that one would obtain a self-consistent solution resulting in a singular matrix. We note that the $\Lambda$-amplitudes are proportional to the complex conjugate $T$-amplitudes to the first order in standard CC with a Hermitian Hamiltonian, $\lambda \sim \tau^{\dagger}$. This is a consequence of the Hamiltonian being Hermitian. If the basis transformation remains close to unitary, we can expect the largest coefficients to appear on the diagonal in $\boldsymbol{A}$ and be positive because these coefficients are the products of matching indices in Eq. (24). In the Appendix, we explore the minimal example of two electrons in two orbitals.

To complete the proof, we must also show that a standard $\mathrm{CC}$ wavefunction rotated to a basis where $\lambda_{1}$ and $\tau_{1}$ are zero would satisfy the equations. We will now investigate the existence and uniqueness of such a rotation using the concept of strong monotonicity. ${ }^{41-43}$ Note that setting $\kappa=\Lambda_{1}-T_{1}$ will remove $\Lambda_{1}$ and $T_{1}$ from the cluster operators to first order in $\boldsymbol{\kappa}$ and the amplitudes. To simplify, we assume that our starting basis is one where $T_{1}$ is zero which can always be reached by setting $\kappa=-T_{1}$. A function $f$ is said to be locally strongly monotone if the function $\Delta\left(\kappa_{1}, \kappa_{2}\right)$ satisfies Eq. (32) for all $\kappa_{1}$ and $\kappa_{2}$ on an open set, $b$,

$$
\begin{aligned}
\Delta\left(\kappa_{1}, \kappa_{2}\right) & =\left\langle f\left(\kappa_{1}\right)-f\left(\kappa_{2}\right), \kappa_{1}-\kappa_{2}\right\rangle \\
& \geq c\left\|\kappa_{1}-\kappa_{2}\right\|^{2} .
\end{aligned}
$$

In Eq. (32), $\langle\cdot, \cdot\rangle$ indicates an inner product, and in this case it is simply the vector product of the vector function $f$ over the compound indices $a i$ and $i a$. By Zarantonello's theorem, the equation $f(x)=a$ has a locally unique solution on $b$ if $f$ is strongly monotone..$^{41}$ The vector function $f$ is of the same size as $\boldsymbol{\kappa}$ and can be divided into two parts, $f_{a i}$ and $f_{i a}$, that are the projections of $T_{1}$ and $\Lambda_{1}$, respectively,

$$
\begin{aligned}
f_{a i} & =\left\langle\tilde{\phi}\left|Y_{i a} \exp (\boldsymbol{\kappa})\right| \Psi\right\rangle, \\
f_{i a} & =-\left\langle\tilde{\Psi}\left|\exp (-\boldsymbol{\kappa}) X_{a i} \exp (\boldsymbol{\kappa})\right| \Psi\right\rangle .
\end{aligned}
$$

By expanding $f_{a i}$ and $f_{i a}$ to first order in $\boldsymbol{\kappa}$, we can write $\Delta$ in quadratic form. If the corresponding matrix is positive definite, Eq. (32) holds

$$
\begin{gathered}
\Delta \approx\left(\Delta \kappa_{a i} \Delta \kappa_{i a}\right)\left(\begin{array}{l}
\boldsymbol{I}\left\langle\tilde{\phi}\left|a_{i}^{\dagger} \tilde{a}_{a} a_{j}^{\dagger} \tilde{a}_{b}\right| \Psi\right\rangle \\
\mathbf{0}
\end{array}\right)\left(\begin{array}{l}
\Delta \kappa_{b j} \\
\Delta \kappa_{j b}
\end{array}\right), \\
\boldsymbol{B}=\boldsymbol{I}-\left\langle\tilde{\Psi}\left|\delta_{i j} a_{a}^{\dagger} \tilde{a}_{b}+\delta_{a b} \tilde{a}_{i} a_{j}^{\dagger}\right| \Psi\right\rangle .
\end{gathered}
$$

The upper left block in Eq. (35) is simply the identity matrix, while the upper right block reduces to $\tau_{i j}^{a b}$. The lower left block is zero, and $\boldsymbol{\kappa}^{u}$ leaves $\lambda_{1}$ unchanged to first order. Finally, the lower right block is the identity plus some additional terms that are at least second order in the amplitudes. Assuming that the reference state dominates the wavefunction, the matrix will be positive definite and $\Delta$ is strongly monotone. Consequently, there must be a unique orbital rotation of the untruncated CC solution for single reference cases that removes $T_{1}$ and $\Lambda_{1}$. Because this wavefunction will satisfy the singles projection by definition, Eq. (15) will also be satisfied and the wavefunction is a solution of the NOCC equations.

In multireference systems, the picture is more complicated. Standard CC explicitly satisfies all the projection equations and has the correct solution. However, it is less clear 
whether it is still possible to rotate away $T_{1}$ and $\Lambda_{1}$. The form of $\Delta$ in Eq. (35) is obtained by approximating the exponential of $\boldsymbol{\kappa}$ with a linear expansion. This will no longer be valid when $T_{1}$ and $\Lambda_{1}$ become large, and $\Delta$ will become a complicated function depending on higher order terms in $\kappa$.

It is worthwhile to briefly compare NOCC and OCC. First, we note that the orbital rotation that removes both $\Lambda_{1}$ and $T_{1}$ from the standard $\mathrm{CC}$ wavefunction is not unitary and the untruncated OCC wavefunction cannot be a solution to the $\mathrm{CC}$ equations in general. Comparing Eqs. (14) and (15) with the OCC equivalent, we see that enforcing a unitary transformation halves the number of transformation parameters and the equivalent of Eqs. (14) and (15) is a single equation,

$$
\begin{aligned}
\frac{\partial L}{\partial \kappa_{\mu_{1}}} & =\left\langle\tilde{\Lambda}\left|\exp (-T)\left[H, X_{\mu_{1}}-Y_{\mu_{1}}\right] \exp (T)\right| \phi\right\rangle \\
& \Rightarrow\left\langle\tilde{\Lambda}\left|\exp (-T)\left[H, X_{\mu_{1}}\right] \exp (T)\right| \phi\right\rangle \\
& =\left\langle\tilde{\Lambda}\left|\exp (-T)\left[H, Y_{\mu_{1}}\right] \exp (T)\right| \phi\right\rangle .
\end{aligned}
$$

This is sufficient to satisfy the conditions of the Ehrenfest theorem, but does not require the terms to be zero on their own. ${ }^{18}$ Consequently, the singles projection equations are not satisfied as noted by Köhn and Olsen. ${ }^{27}$ For NOCC, the two terms are zero independently.

\section{CONCLUSION}

In this contribution, we have demonstrated that NOCC is equivalent to FCI in the untruncated limit for chemical systems under some assumptions. In particular, the eigenfunction of the Hamiltonian will satisfy the NOCC equations. However, it is not possible to demonstrate that this solution is unique in general. In principle, Hamiltonians can be constructed that makes the $\boldsymbol{A}$-matrix in Eq. (28) singular, but this seems highly unlikely to occur in chemical systems. Interestingly, truncated NOCC does not satisfy the singles projection equations because the second term in Eq. (19) and the right-hand side in Eq. (20) are not zero. The same is true for OCC, and it does not appear to have a large effect at least in well-behaved systems. ${ }^{19,21}$

The advantage of NOCC compared to standard CC is that it is inherently gauge invariant, assuming a gauge invariant basis. While the effect of gauge dependence is usually small for most properties in CC theory, magnetic properties become origin dependent, resulting in unphysical behavior. This is very unsatisfying, especially when considering that $\mathrm{CC}$ is often the most accurate method available and used for benchmark calculations.

Truncated NOCC is unlikely to improve much on standard $\mathrm{CC}$ with respect to a multireference system except for special cases. However, most multireference $\mathrm{CC}$ methods involve some sort of active space. This is especially true for single reference-based multireference methods. ${ }^{10}$ Obtaining a good active space is critical in such methods, and NOCC type orbital transformations makes it possible to include the transformation of the active space consistently in the Lagrangian. It would also be very interesting to see how the paired $\mathrm{CC}$ methods perform using NOCC orbitals instead of orthogonally optimized orbitals.

Finally, it is worth noting that NOCC can be viewed as a special case of OACC where the entire orbital space is included in the active space. ${ }^{38}$ Orbital adapted CC makes it possible to obtain an optimal subspace of the Hilbert space spanned by the basis set. Basis set incompleteness is often the limiting factor for the accuracy of CC calculations, and the OACC approach might make it possible to obtain greater accuracy at the same computational cost.

\section{ACKNOWLEDGMENTS}

I would like to thank Simen Kvaal, Henrik Koch, Thomas Bondo Pedersen, Eirik Kjønstad, and Fabian Faulstich for valuable discussions and input to this project. This work has received funding from No. ERC-STG-2014 under Grant No. 639508 and from the Research Council of Norway through its Centres of Excellence scheme, Project No. 262695.

\section{APPENDIX: MINIMAL EXAMPLE}

In this appendix, we will investigate the minimal example of two electrons in four spin orbitals. In chemistry, this corresponds to the hydrogen molecule in a minimal basis and we will assume a real symmetric Hamiltonian. Because the Hamiltonian does not couple singlet and triplet states, we do not have to worry about the triplet states if we assume our reference states are singlets because the cluster operators are reduced to a single singlet double excitation and its associated amplitude,

$$
\begin{array}{r}
\left\langle\tilde{\phi}\left|=\left\langle-\left|\tilde{a}_{1 \alpha} \tilde{a}_{1 \beta} \quad\right| \phi\right\rangle=a_{1 \beta}^{\dagger} a_{1 \alpha}^{\dagger}\right|-\right\rangle, \\
\Lambda=\lambda a_{1 \alpha}^{\dagger} a_{1 \beta}^{\dagger} \tilde{a}_{2 \beta} \tilde{a}_{2 \alpha} \quad T=\tau a_{2 \alpha}^{\dagger} a_{2 \beta}^{\dagger} \tilde{a}_{1 \beta} \tilde{a}_{1 \alpha} .
\end{array}
$$

Furthermore, products of the $T$-operator will be at least a quadruple excitation, so both the right and left wavefunctions will be linear in $T$, greatly simplifying the Lagrangian

$$
\begin{aligned}
L & =\langle\tilde{\Psi}|H| \Psi\rangle \\
& =\langle\tilde{\phi}|(1+\Lambda) \exp (-T) H \exp (T)| \phi\rangle \\
& =\langle\tilde{\phi}|(1+\Lambda)(1-T) H(1+T)| \phi\rangle .
\end{aligned}
$$

Taking the derivative of $L$ with respect to $\lambda$ gives us the equation for the amplitude $\tau$, resulting in a second order polynomial equation,

$$
\begin{aligned}
\frac{\partial L}{\partial \lambda} & =\left\langle\tilde{\mu}_{2}|(1-T) H(1+T)| \phi\right\rangle \\
& =\langle\tilde{\phi}|H| \phi\rangle+\tau\left\langle\tilde{\mu}_{2}|H| \mu_{2}\right\rangle-\tau\langle\phi|H| \phi\rangle-\tau^{2}\left\langle\phi|H| \mu_{2}\right\rangle \\
& =H_{20}+\tau\left(H_{22}-H_{00}\right)-\tau^{2} H_{02}=0, \\
\tau & =\frac{\left(H_{22}-H_{00}\right) \pm \sqrt{\left(H_{22}-H_{00}\right)^{2}+4 H_{02} H_{20}}}{2 H_{02}}
\end{aligned}
$$

If $H$ was symmetric, we would have $H_{02}=H_{20}$ and the amplitude would be real, given a real Hamiltonian. This is no longer guaranteed with a biorthogonal basis because $H_{02}$ and $H_{20}$ can differ and, in principle, even have different signs. This seems unlikely to happen if we consider two cases of the hydrogen molecule: equilibrium bond length and the two atoms 
infinitely far apart. In the equilibrium case, the wavefunction is dominated by a single reference and the orbitals will be close to the canonical molecular orbitals. In such a case, $\mathrm{H}_{02} \mathrm{H}_{20}$ will be positive and the two roots will correspond to the ground and excited state of the molecule. Because the terms under the square root are greater than the orbital difference, $H_{22}-H_{00}$, the overall sign of the amplitude will depend on the choice of the root and $H_{02}$. Note that the magnitude of $\tau$ goes toward 0 or $\infty$ as the difference in orbital energies increases, depending on the choice of root. In the infinitely stretched case, the orbital difference is zero and the expression is simplified. Again, the overall sign depends on the choice of root and $H_{02}$ and a symmetric matrix results in $\tau=1$.

Solving for $\lambda$ results in a linear equation that depends on $\tau$,

$$
\begin{aligned}
\frac{\partial L}{\partial \tau}= & \left\langle\tilde{\phi}|(1+\Lambda)(1-T) H| \mu_{2}\right\rangle-\lambda\langle\tilde{\phi}|H(1+T)| \phi\rangle \\
= & \left\langle\tilde{\phi}|H| \mu_{2}\right\rangle+\lambda\left\langle\tilde{\mu}_{2}|H| \mu_{2}\right\rangle-\lambda \tau\left\langle\tilde{\phi}|H| \mu_{2}\right\rangle \\
& -\lambda\langle\tilde{\phi}|H| \phi\rangle-\lambda \tau\left\langle\tilde{\phi}|H| \mu_{2}\right\rangle \\
= & H_{02}+\lambda\left(H_{22}-H_{00}\right)-2 \lambda \tau H_{02}=0 .
\end{aligned}
$$

Inserting the expression for $\tau$, we again obtain an expression where the sign depends on the choice of root and $H_{02}$. Due to the intermediate normalization, the magnitude of $\lambda$ will always go to 0 when the difference in orbital energies increases and will go to $\frac{1}{2}$ when the orbitals become degenerate and the Hamiltonian is symmetric,

$$
\lambda=\frac{H_{02}}{2 \tau H_{02}-\left(H_{22}-H_{00}\right)}=\frac{H_{02}}{ \pm \sqrt{\left(H_{22}-H_{00}\right)^{2}+4 H_{02} H_{20}}} .
$$

Importantly, the sign of $\lambda \tau$ will always be positive if $H_{02} H_{20}$ is positive,

$$
\lambda \tau=\frac{\left(H_{22}-H_{00}\right) \pm \sqrt{\left(H_{22}-H_{00}\right)^{2}+4 H_{02} H_{20}}}{ \pm 2 \sqrt{\left(H_{22}-H_{00}\right)^{2}+4 H_{02} H_{20}}} .
$$

Finally, the $\boldsymbol{A}$ matrix from Eq. (28) becomes diagonal in this system. This can be realized by noting that there are no indices left to sum over in Eqs. (25) and (26) for the off-diagonal elements,

$$
A_{a i, a i}=1+2 \lambda \tau=2 \pm \frac{\left(H_{22}-H_{00}\right)}{\sqrt{\left(H_{22}-H_{00}\right)^{2}+4 H_{02} H_{20}}} .
$$

Strictly speaking, there is no guarantee that $\mathrm{H}_{02} \mathrm{H}_{20}$ is positive in the biorthogonal basis. However if the basis consists of two hydrogenic orbitals, the non-Hermitian terms in $H_{02}$ and $H_{20}$ will be at least second order in $\kappa$. Furthermore, in the case of infinitely stretched bond length, $A_{a i, a i}=2$, unless $H_{02} H_{20}$ also goes to zero somehow.
${ }^{1}$ R. J. Bartlett and M. Musial, Rev. Mod. Phys. 79, 291 (2007).

${ }^{2}$ J. Olsen and P. Jørgensen, J. Chem. Phys. 82, 3235 (1985).

${ }^{3}$ H. Koch and P. Jørgensen, J. Chem. Phys. 93, 3333 (1990).

${ }^{4}$ J. F. Stanton and R. J. Bartlett, J. Chem. Phys. 98, 7029 (1993).

${ }^{5}$ S. Saebø and P. Pulay, Annu. Rev. Phys. Chem. 44, 213 (1993).

${ }^{6}$ T. D. Crawford, "Reduced-scaling coupled-cluster theory for response properties of large molecules," in Recent Progress in Coupled Cluster Methods: Theory and Applications, edited by P. Cársky, J. Paldus, and J. Pittner (Springer Netherlands, Dordrecht, 2010), pp. 37-55.

${ }^{7}$ M. Schwilk, Q. Ma, C. Köppl, and H.-J. Werner, J. Chem. Theory Comput. 13, 3650 (2017).

${ }^{8}$ T. Kjærgaard, P. Baudin, D. Bykov, K. Kristensen, and P. Jørgensen, Wiley Interdiscip. Rev.: Comput. Mol. Sci. 7, e1319 (2017).

${ }^{9}$ A. Köhn, M. Hanauer, L. A. Mück, T.-C. Jagau, and J. Gauss, Wiley Interdiscip. Rev.: Comput. Mol. Sci. 3, 176 (2013).

${ }^{10}$ D. I. Lyakh, M. Musiał, V. F. Lotrich, and R. J. Bartlett, Chem. Rev. 112, 182 (2012).

${ }^{11}$ T. B. Pedersen and H. Koch, J. Chem. Phys. 106, 8059 (1997).

${ }^{12}$ T. B. Pedersen and H. Koch, Chem. Phys. Lett. 293, 251 (1998).

${ }^{13}$ M. C. Tam, N. J. Russ, and T. D. Crawford, J. Chem. Phys. 121, 3550 (2004).

${ }^{14}$ T. D. Crawford and P. J. Stephens, J. Phys. Chem. A 112, 1339 (2008).

${ }^{15}$ T. B. Pedersen, H. Koch, L. Boman, and A. M. Sánchez de Merás, Chem. Phys. Lett. 393, 319 (2004).

${ }^{16}$ F. London, J. Phys. Radium 8, 397 (1937).

${ }^{17}$ R. Ditchfield, Mol. Phys. 27, 789 (1974).

${ }^{18}$ T. B. Pedersen, H. Koch, and C. Hättig, J. Chem. Phys. 110, 8318 (1999).

${ }^{19}$ T. B. Pedersen, B. Fernández, and H. Koch, J. Chem. Phys. 114, 6983 (2001).

${ }^{20}$ G. E. Scuseria and H. F. Schaefer, Chem. Phys. Lett. 142, 354 (1987).

${ }^{21}$ C. D. Sherrill, A. I. Krylov, E. F. C. Byrd, and M. Head-Gordon, J. Chem. Phys. 109, 4171 (1998).

${ }^{22}$ K. A. Brueckner, Phys. Rev. 96, 508 (1954).

${ }^{23}$ R. A. Chiles and C. E. Dykstra, J. Chem. Phys. 74, 4544 (1981).

${ }^{24} \mathrm{H}$. Koch, R. Kobayashi, and P. Jørgensen, Int. J. Quantum Chem. 49, 835 (1994).

${ }^{25}$ F. Coester, Nucl. Phys. 7, 421 (1958).

${ }^{26}$ U. Bozkaya, J. M. Turney, Y. Yamaguchi, H. F. Schaefer III, and C. D. Sherrill, J. Chem. Phys. 135, 104103 (2011).

${ }^{27}$ A. Köhn and J. Olsen, J. Chem. Phys. 122, 084116 (2005).

${ }^{28}$ H. R. McAlexander, T. J. Mach, and T. D. Crawford, Phys. Chem. Chem. Phys. 14, 7830 (2012).

${ }^{29}$ J. Cullen, Chem. Phys. 202, 217 (1996).

${ }^{30}$ T. Stein, T. M. Henderson, and G. E. Scuseria, J. Chem. Phys. 140, 214113 (2014).

${ }^{31}$ T. M. Henderson, I. W. Bulik, T. Stein, and G. E. Scuseria, J. Chem. Phys. 141, 244104 (2014).

32 J. A. Parkhill, K. Lawler, and M. Head-Gordon, J. Chem. Phys. 130, 084101 (2009).

${ }^{33}$ S. Lehtola, J. Parkhill, and M. Head-Gordon, J. Chem. Phys. 145, 134110 (2016).

${ }^{34}$ S. Kvaal, Mol. Phys. 111, 1100 (2013).

${ }^{35}$ J. Arponen, Ann. Phys. 151, 311 (1983).

${ }^{36}$ J. Arponen and R. Bishop, Ann. Phys. 207, 171 (1991).

${ }^{37}$ A. Laestadius and S. Kvaal, "Analysis of the extended coupled-cluster method in quantum chemistry," SIAM J. Numer. Anal. (to be published); e-print arXiv:1702.04317 [math.NA] (2017).

${ }^{38}$ S. Kvaal, J. Chem. Phys. 136, 194109 (2012).

${ }^{39}$ P.-O. Löwdin, Phys. Rev. 97, 1490 (1955).

${ }^{40}$ R. Balian and E. Brezin, Nuovo Cimento B 64, 37 (1969).

${ }^{41}$ E. H. Zarantonello, MRC Technical Summary Report (Mathematics Research Center, 1960), p. 160.

${ }^{42}$ E. Zeidler, Nonlinear Functional Analysis and Its Applications, 1st ed. (Springer-Verlag New York, New York, 1990).

${ }^{43}$ T. Rohwedder, ESAIM: Math. Modell. Numer. Anal. 47, 421 (2013). 